inches, between the anterior superior spines of the essential portion of the omentum (the greatthe ilia $7 \frac{1}{2}$ inches, between the trochanters $12 x / 2$, er omentum alone is referred to in this paper) and the external conjugate diameter being $61 / 4$ consists of peritoneum, and that its function, by inches. Under full anæsthesia the dilatation of virtue of the abundance of fat which forms one the os uteri was completed normally. The occiput was rotated from left-posterior to left-anterior position, and, without removal of the hand, the forceps was applied. Traction-rods were attached. After tremendous pulling, a male infant weighing 8 lbs. was delivered. His right eyelid was torn. With some difficulty he was resuscitated, but he died the next day, evidently from injuries received during delivery. The mother's vagina suffered several slight lacerations.

Case 3.-Mrs. H., quartipara, 29 years old, after several hours of ineffectual second-stage labor, was etherized, Dr. H. A. Wood assisting, March 24, I89r. The position of the foetus was occipito-right-posterior. With the whole hand inside the uterus, the position was changed to occipito-anterior, and forceps applied before withdrawing the hand. A male child weighing io lbs. was then easily delivered. The mother's vagina and perineum suffered no damage.

Case 4.-Mrs. C., primipara, 22 years old, had been in labor twenty-four hours, and the pains had almost ceased, when, with Dr. H. A. Wood's assistance, she was etherized. The fotal head had descended to the inferior straits in left posterior position. In spite of good flexion, there had been no sign of natural rotation. The head was thereupon pushed back above the pelvic brim, rocated to anterior position, and forceps applied to it before removing my hand. Extraction was then easy. The female child weigbed 7 lbs. and was uninjured, although one blade of the forceps was applied to the right eyebrow. The mother was also uninjured.

\title{
THE MANAGEMENT OF THE OMENTUM IN ABDOMINAL OPERATIONS.
}

Read in the Section of Obstetrics and Diseases of Women, at the Forty second Annual Meeting of the American Medical Association, held at Washington, D. C., May, $189 \mathrm{I}$.

BY ANDREW E. CURRIER, M.D., OF NEW YORK CITY.

It is hoped that it will not be considered impertinent to offer a few suggestions upon this subject, apparently so simple, before a body composed so largely of abdominal surgeons, or surgeons who are familiar with abdominal operations, as is this section. The fact has been impressed upon my mind not only by my own experience, but by observation of the work of others, and a search of a considerable portion of the literature of abdominal surgery, that the subject in question has not received the attention which it deserves, and that it is by no means a matter of insignificance. This statement will be the more readily accepted if one remembers that of its constituent elements, is of importance, inasmuch as it protects to a greater or less extent, the viscera which lie under it from sudden changes of temperature. Such a covering is therefore of great value in those localities in which climatic changes are not only sudden but severe, and in which one requires all the resources of art as well as nature to preserve the physical equilibrium.

It is therefore desirable in all cases in which the abdominal cavity is exposed that one observe the following propositions with reference to the omentum:

1. It should be preserved as nearly intact as the conditions of each individual case will allow.

2. Great care should be exercised to avoid wounding it in making the abdominal incision, or bruising or lacerating it at subsequent stages of an operation.

3. Badly injured portions should be resected with all due precautions, also such portions as cannot be so replaced as to occupy their original position and perform their normal function.

4. Before closing the abdominal wound it should be carefully and evenly replaced as the natural covering of the intestine.

In the large class of cases in which the lesions demanding surgical interference are located entirely, or to a great extent, in the pelvis, the omentum is very frequently a source of no particular trouble during an operation. If the incision is a short.one with its lower limit near the symphysis pubis, and the omentum is not voluminous or adberent it may not be seen at all, and we are conscious of its presence only as it becomes entangled about the fingers when they are passed downward into the pelvis. The operation may be completed and the incision closed without a thought or an intimation of the presence of an omentum.

If an abdominal sponge is used to absorb the leakage from divided vessels and protect the intestines it must not be forgotten that as the sponge presses the latter upward it presses the omentum upward also, and when the sponge is withdrawn the omentum may still remain displaced, or gathered into a lump, or otherwise $a b-$ normally disposed so that it can no longer perform its proper function. In all the secondary abdominal sections which I have seen, including one of my own, I have never seen the omentum in its normal position. In my case neglect to properly replace this structure furnished opportunity for adhesion of the intestine to the parietal peritoneum, and so near was it to the incision that it barely escaped injury from the knife. In those cases in which the omentum is normal in 
appearance but adherent to the parietal or visceral peritoneum the necessity for careful management must be self-evident. If it has been roughly handled peritonitis with new adhesions will almost certainly result. It must therefore be so evenly and so carefully disposed that if new adhesions are formed they will cause the least possible disturbance to the functional activity of the viscera to which they are attached.

Another class of cases includes those in which the omentum is atrophied or defective.

If the abdominal cavity is encroached upon by a large tumor, whether cystic or solid, the integrity of the omentum and its functional usefulness are generally destroyed. With such growths inflammatory processes are of frequent occurrence and may result in the most intimate adhesive union of the omentum with the peritoneal covering of the tumor. Should the new growth attain a large size and the omentum be imprisoned between it and the abdominal wall, the resulting pressure would cause absorption and disappearance of more or less of the fatty tissue in the omental structure, though the adhesive union of the peritoneal surfaces might continue. Of course it may happen that the omentum will be pushed upward by the advancing tumor, and that disturbances from pressure or inflammation will not be of such a character as to impair its structure. If atrophy have resulted from this or any other cause -by atrophy being meant the condition in which the quantity of omental fat is very small, it is not an indication that the omentum will require less careful treatment; on the contrary it must be handled with the greatest delicacy, and spread over the intestine with great nicety before the abdominal wound is closed, for the frailty of its structure endangers its integrity, and a small rent which might be overlooked might furnish entrance for a loop of intestine with disastrous strangulation as a consequence.

In the removal of large tumors with adherent omentum, conservatism and safety alike demand the free excision of all strips and fragments of the destroyed and useless structure. Too often has it been the method to tear away adherent portions from the tumor, tie their bleeding points, and then drop them, careless as to their arrangement, and forgetful of the fact that attachment to the intestinal may result in such a way as to favor intestine obstruction. A far better plan seems to me to leave no long ends floating about among the intestines, but to cut them off as high up as possible, even if it be necessary to approach the neighborhood of the stomach, then having obtained a straight or nearly straight lower border, to tie all bleeding points and sear the entire edge with the cantery at a low temperature.

Another class of cases, which, however, is a small one, includes those in which the omentum is hypertrophied. Such cases may be very trouble- some. Not only is the deposit of fat of great thickness, but the structure may extend, like a long apron, deeply into the pelvis. To replace such a structure properly is a very difficult matter unless the abdominal incision has been a very long one, and even then it may not be entirely successful. Such a case recently occurred in my practice. The patient was a young widow, a masseuse by occupation, who was recovering from peritonitis and metritis following a curettement of the uterus, when $I$ was called to see her in consultation. In addition to these inflammatory troubles there was a distinct tumor on either side of the uterus, within the pelvis. The patient was hysterical and a morphine eater, but an abdominal operation did not promise to be extraformidable or extra-hazardous. The adhesions in the pelvis were readily broken up, an ovarian tumor as large as a hen's egg, with adherent and dilated tube, was removed from the left side, and equally degenerated but not quite so large appendages from the right side. The omentum dipped deeply into the pelvis and particular pains were taken to tuck it carefully and smoothly down. The effort was unsuccessful, for the patient died on the sixth day from septic general peritonitis (though there were also two perforations on the anterior aspect of the descending colon), and the inferior portion of the omentum on the right side was compressed into a mass as large as a child's fist, which was necrotic, evidently from interference with its circulation. I do not know that this was the primary or principal factor in producing the fatal result, but it was a factor and therefore carries a lesson and a warning with it. It would have been better in this case, and the rule would apply in all similar cases, to excise the lowest fourth of the omentum throughout its entire width. The remainder would have furnished ample covering for the intestines, and the bad results from pressure might have been obviated. The case which has been narrated is also illustrative of an accident to which the omentum is susceptible when hypertrophied or when adherent to the parietal peritoneum. In the case in question the omentum was very thick and in contact with but not adherent to the parietal peritoneum. In making the incision the tissues below the recti muscles were cut layer by layer, being made tense by the traction of hæmostatic forceps secured to either side of the wound, and then diviced. Such a plan usually enables one to avoid cutting either omentum or intestine, for as soon as the peritoneal cavity is reached the tension of the forceps on either side of the wound draws its edges apart and reveals the space. It failed to do so in this instance and the omentum was incised on the supposition that the præ-peritoneal fat was being cut. Of course the mistake was soon discovered and with it the fact that the omentum was hyper- 
trophied not only as to thickness but also as to length. I know of no infallible rule for preventing such accidents. It has never happened to me before and I can conceive of its happening to even the most careful and expert operators.

Wounds of the omentum may become sources of danger from sepsis, peritonitis, or hæmorrhage. In cases in which the omentum has been greatly torn or bruised sepsis and peritonitis are probably more frequent than is generally supposed. Certainly a loosely constructed, freely absorbing, easily degenerating tissue like this offers favorable conditions for serious inflammatory and septic trouble when once an injury has been received. The subject is one which invites pathological investigation in unexplored territory. Hæmorrhage from the omentum may be troublesome, serious or fatal. It is more likely to be venous than arterial, an oozing which may show little tendency to cease. A case of hæmorrhage of this character is reported as recently as May i 8, of this year, in the New York Medical Journal, and in the practice of so eminent and careful a surgeon as Prof. William T. Bull. Fortunately in this case the bleeding was relieved by means of an iodoform gauze tampon within the abdomen, but such treatment would be ineffective and haphazard in many cases and might do serious damage by pressure upon other structures. Hæmorrhage from the omentum can usually be con trolled by ligating the layer vessels, whether veins ot arteries, with fine silk and touching all oozing points with the cautery. The latter is more effective than hot water irrigation, especially if trouble is anticipated from many oozing points.

Rents and incisions in the omentum have already been referred to, and the peculiar dangers to which they give rise. Hence they must not be neglected nor slighted whether small or large. They should be carefully and accurately closed with a continuous suture of fine Chinese silk or reliable catgut. It should not be forgotten by the abdominal surgeon that the object of his art is not merely to relieve the present suffering of a patient and remove an existing lesion, but to avoid the induction of other lesions which might be more intolerable than the original one. Unfortunately, such an ideal result is not always possible even to the most skillful or the most cautious, and this fact warrants constant vigi. lance and unwearying discussion of the subject in all its bearings and possibilities.

159 E. 37 th St.

IN HoNOR OF VIRCHOW.-The freedom of the City of Berlin is to be extended to the eminent pathologist and medical patriarch on his seventieth birthhay; and upon the same occasion his portrait will be hung in the Council Chamber.

\section{THE REMOTE EFFECTS OF FGTAL,}

BRAIN INJURY IN LABOR; OR, WHY WE ARE RIGHTHANDED.

Read in the Section of Obstetrics and Diseases of Women, at the Fortysecond Annual Meeting of the American Medical Association, held

at Washington, D. C., May, 1897 .

BY F. W. GOODALL, M.D., OF BENNINGTON, VT.

We are right-handed because of injury of the motor centres of the right side of the fotal brain in labor. The manner of receiving this injury, and something of its nature, constitute the subject of this paper.

Some may take exceptions to my premises, and assert that the causes which determine the position of the fotus "at term," are the primary causes of right-handedness. This I decline to discuss. Much might be said concerning the bones, diameters, planes and outlet of the female pelvis, in introducing my subject. All these points I pass over, and pause only to say that the contour of the parts, or the natural structure and relations of the various bones and organs pertaining to the female pelvis, in connection with the physical character of the foetus, determines in a mechanical way how it shall present at delivery, and the presentation determines what motor centres, if any, shali be injured ; and for the purposes of this paper, I embrace all head presentations under four varieties, which will be briefly defined in passing. I deal only with the first and fourth varieties, as it is only in these presentations that the right side of the head takes the brunt of labor. Largely in consequence of the rectum occupying the notch in the left side of the sacrum, the fotal head, which is naturally the presenting part, presents, in the great majority of cases, with its long axis in the right oblique diameter, the occiput being directed towards the left acetabulum, and the forehead towards the right sacro-iliac symphysis. From the relative position of the uterus, the pelvic canal, its floor and outlet, taken in connection with this position of the fotal head, the most advanced point of the head in the progress of delivery is usually somewhere to the right of a line drawn from the nasion (root of the nose) to the inion (base of the external occipital protuberance), and between the anterior and posterior fontanelles, a very important field of motor centres. In this position of the head, which is called in obstetric language the "first position," its long diameter is nearly parallel with the superior plane of the pelvis at the commencement of labor, but there is a very decided lateral obliquity of the head, in consequence of the inclination forwards and downwards of the fundus uteri, the right side of the cranium being considerably lower than the left, and the most depending part of the cranium is the right parietal eminence. 